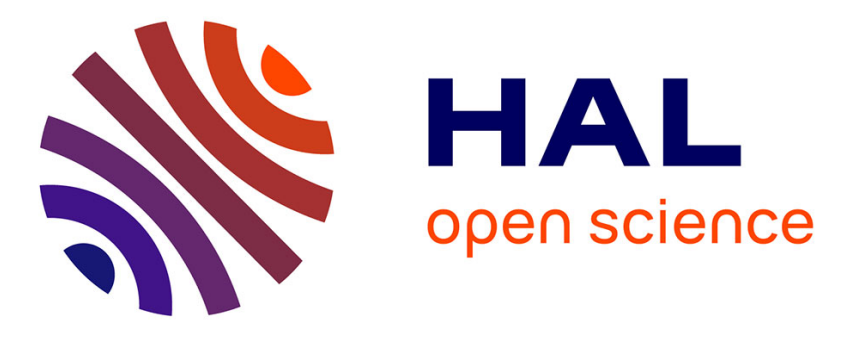

\title{
Asymptotic-preserving Godunov-type numerical schemes for hyperbolic systems with stiff and non-stiff relaxation terms
}

Christophe Berthon, Christophe Chalons, Rodolphe Turpault

\section{To cite this version:}

Christophe Berthon, Christophe Chalons, Rodolphe Turpault. Asymptotic-preserving Godunov-type numerical schemes for hyperbolic systems with stiff and non-stiff relaxation terms. Numerical Methods for Partial Differential Equations, 2012, vol 29 (4), pp 1149-1172. 10.1002/num.21749 . hal-00909103

\section{HAL Id: hal-00909103 \\ https://hal.science/hal-00909103}

Submitted on 25 Nov 2013

HAL is a multi-disciplinary open access archive for the deposit and dissemination of scientific research documents, whether they are published or not. The documents may come from teaching and research institutions in France or abroad, or from public or private research centers.
L'archive ouverte pluridisciplinaire HAL, est destinée au dépôt et à la diffusion de documents scientifiques de niveau recherche, publiés ou non, émanant des établissements d'enseignement et de recherche français ou étrangers, des laboratoires publics ou privés. 


\title{
Asymptotic-preserving Godunov-type numerical schemes for hyperbolic systems with stiff and non-stiff relaxation terms
}

\author{
C. Berthon, C. Chalons and R. Turpault
}

March 28, 2012

\begin{abstract}
We devise a new-class of asymptotic-preserving Godunov-type numerical schemes for hyperbolic systems with stiff and non-stiff relaxation source terms governed by a relaxation time $\varepsilon$. As an alternative to classical operator-splitting techniques, the objectives of these schemes are twofold: first, to give accurate numerical solutions for large, small and in-between values of $\varepsilon$ and second, to make optional the choice of the numerical scheme in the asymptotic regime $\varepsilon$ tends to zero. The latter property may be of particular interest to make easier and more efficient the coupling at a fixed spatial interface of two models involving very different values of $\varepsilon$.
\end{abstract}

\section{Introduction}

In the present work, we consider the numerical approximation of hyperbolic systems with relaxation-type source-term in the following form:

$$
\begin{aligned}
\partial_{t} u+\partial_{x} v & =0, \\
\partial_{t} v+\partial_{x} g(u, v) & =\frac{1}{\varepsilon(t, x, u)} S(u, v) .
\end{aligned}
$$

The state vector $W=(u, v)^{\top}$ belongs to $\Omega$, a convex subset of $\mathbb{R}^{2}$. The function $g: \Omega \rightarrow \mathbb{R}$ is assumed to be smooth enough and we impose the system to be strictly hyperbolic. For the sake of simplicity in the notations, we set $G(W)=(v, g(u, v))^{\top}$ the flux function of the system and we note $\lambda_{1}(u, v)<\lambda_{2}(u, v)$ the eigenvalues of the Jacobian matrix $\nabla_{W} G(W)$.

Concerning the source term, we adopt the well-known formalism of ChenLevermore-Liu [12]. More precisely, we assume the existence of a unique equilibrium $v=f(u)$ such that $S(u, f(u))=0$ to define a relaxed model:

$$
\partial_{t} u+\partial_{x} f(u)=0 .
$$


As usual, the stability of this local equilibrium solution is satisfied under the Whitham subcharacteristic condition:

$$
\left|f^{\prime}(u)\right|<\min \left(\left|\lambda_{1}(u, v)\right|,\left|\lambda_{2}(u, v)\right|\right), \forall(u, v) \in \Omega .
$$

For instance, choosing $S(u, v)=f(u)-v$, one obviously recovers the well-known relaxation model [20]:

$$
\begin{aligned}
\partial_{t} u+\partial_{x} v & =0, \\
\partial_{t} v+\partial_{x} g(u, v) & =\frac{1}{\varepsilon(t, x, u)}(f(u)-v),
\end{aligned}
$$

where $\varepsilon$ is a smooth function of $t, x$ and $u$ which can be considered as a relaxation time. The system (1)-(2) admits two different asymptotic regimes depending on the values of $\varepsilon$. Whenever $\varepsilon$ is small, the system (5)-(6) enters the framework of Chen-Levermore-Liu [12] to relax to (3). On the other hand, as soon as $\varepsilon$ is large, (5)-(6) simply becomes the following homogeneous transport system:

$$
\begin{aligned}
\partial_{t} u+\partial_{x} v & =0, \\
\partial_{t} v+\partial_{x} g(u, v) & =0 .
\end{aligned}
$$

Our objective is to derive a numerical scheme able to produce a relevant approximation of (1)-(2) in every regime. Since we are interested in general forms of $\varepsilon$, it is crucial that our numerical scheme behaves correctly in both asymptotic regimes as well as for transitional regimes which occur for in-between values of $\varepsilon$.

During the last decade, several asymptotic-preserving schemes were proposed in the literature and we refer for instance to $[10,17,13,14,9,7,6,11]$ for some of the most recent ones, but also to the references proposed therein. These schemes are able to give a suitable numerical approximation in the limit of both $\varepsilon$ large and small. However, the approximations of each of these asymptotic regimes are closely linked: the choice of the scheme in one regime generally impose the form of the other. Our goal is to develop a technique that is general in the sense that any scheme can be adopted in each limit regime. In addition, the resulting procedure is expected to be accurate in transitional regimes and conserve the usual robustness properties.

Several applications of such a framework can be found in the literature. For instance, the widely studied problem of domain decomposition where $\varepsilon$ is fixed, discontinuous or with a stiff transition around an interface [19]. Here, the goal is generally to reduce the complexity of the model by shifting from the relaxation to the relaxed systems. Another application is devoted to model reduction [22], where the relaxed model is used in numerical simulations whenever a suitable criterion is reached. In theses applications, $\varepsilon$ may depend on the variables. To conclude this non-exhaustive overview of applications, let us mention the coupling between codes where $\varepsilon$ governs the transition from one code to another $[2,1,3]$. The reader is also referred to $[5,23]$ for related works. 
The paper is focused on numerical issues, however the theory of systems of the form (1)-(2) has been carried on in [6].

The paper is organized as follows: in the next section, we briefly recall the notion of consistency of simple approximate Riemann solvers for convective systems with sources. Then, we first introduce our numerical technique for linear transport. We show that the resulting method possesses the correct asymptotic limits and admits a maximum principle. Furthermore, these results are obtained for a standard CFL condition that does not depend on $\varepsilon(t, x, u)$.

The numerical method is then extended to the nonlinear cases (5)-(6) in the third section and (1)-(2) in the fourth section. Similar properties are obtained. Finally numerical simulations are performed to emphasize the relevance of the scheme.

\section{Consistency of simple approximate Riemann solvers and Godunov-type methods}

In this section, we briefly recall the notion of consistency with the integral form for a simple approximate Riemann solver associated with a convective system with sources

$$
\partial_{t} W+\partial_{x} G(W)=S(W)
$$

and initial data

$$
W(x, 0)=\left\{\begin{array}{lll}
W_{L} & \text { if } \quad x<0 \\
W_{R} & \text { if } \quad x>0
\end{array}\right.
$$

We follow Gallice [15], and also refer to [11,4]. Throughout this section, $S$ plays the role of $S / \varepsilon$ in system (1) of previous section.

Let us first recall that a simple approximate Riemann solver takes the following form

$$
W_{\Delta}\left(\frac{x}{t} ; W_{L}, W_{R}\right)=\left\{\begin{array}{l}
W_{1}=W_{L}, \quad \frac{x}{t}<\sigma_{1}, \\
W_{k}, \quad \sigma_{k-1}<\frac{x}{t}<\sigma_{k}, \quad k=2, . ., m, \\
W_{m+1}=W_{R}, \quad \frac{x}{t}>\sigma_{m}
\end{array}\right.
$$

and thus consists of $m$ waves with speeds $\sigma_{k}=\sigma_{k}\left(W_{L}, W_{R}\right), 1 \leq k \leq m$, and $m-1$ intermediate states $W_{k}, 2 \leq k \leq m$, which may depend on the spacetime mesh step $\Delta=(\Delta x, \Delta t)$. Before defining the notion of consistency in the integral sense, notice that it is natural to require

$$
\lim _{\substack{W_{L}, W_{R} \rightarrow W \\ \Delta \rightarrow 0}} W_{\Delta}\left(\frac{x}{t} ; W_{L}, W_{R}\right)=W
$$

for any $W \in \Omega$. 
Consistency with the integral form of (9). We first observe that under the CFL condition

$$
\max _{1 \leq k \leq m}\left|\sigma_{k}\right| \frac{\Delta t}{\Delta x} \leq \frac{1}{2}
$$

we have

$$
\frac{1}{\Delta x} \int_{-\frac{\Delta x}{2}}^{\frac{\Delta x}{2}} W_{\Delta}\left(\frac{x}{\Delta t} ; W_{L}, W_{R}\right) d x=\frac{1}{2}\left(W_{L}+W_{R}\right)-\frac{\Delta t}{\Delta x} \sum_{k=1}^{m} \sigma_{k}\left(W_{k+1}-W_{k}\right)
$$

so that

$$
\int_{0}^{\Delta t} \int_{-\frac{\Delta x}{2}}^{\frac{\Delta x}{2}} \frac{\partial}{\partial t} W_{\Delta}\left(\frac{x}{t} ; W_{L}, W_{R}\right) d x d t=-\Delta t \sum_{k=1}^{m} \sigma_{k}\left(W_{k+1}-W_{k}\right) .
$$

Integrating (9) in space and time leads to

$$
\int_{0}^{\Delta t} \int_{-\frac{\Delta x}{2}}^{\frac{\Delta x}{2}} \frac{\partial}{\partial t} W d x d t+\int_{0}^{\Delta t} \int_{-\frac{\Delta x}{2}}^{\frac{\Delta x}{2}} \frac{\partial}{\partial x} G(W) d x d t=\int_{0}^{\Delta t} \int_{-\frac{\Delta x}{2}}^{\frac{\Delta x}{2}} S(W) d x d t
$$

which gives, focusing ourselves on simple approximate Riemann solutions of the form (10),

$$
\int_{-\frac{\Delta x}{2}}^{\frac{\Delta x}{2}} W\left(\frac{x}{\Delta t} ; W_{L}, W_{R}\right) d x=\frac{\Delta x}{2}\left(W_{L}+W_{R}\right)-\int_{0}^{\Delta t} \int_{-\frac{\Delta x}{2}}^{\frac{\Delta x}{2}}\left\{\frac{\partial}{\partial x} G(W)-S(W)\right\} d x d t .
$$

Consistency in the integral sense consists in imposing

$$
\int_{0}^{\Delta t} \int_{-\frac{\Delta x}{2}}^{\frac{\Delta x}{2}}\left\{\frac{\partial}{\partial x} G(W)-S(W)\right\} d x d t \approx \Delta t \sum_{k=1}^{m} \sigma_{k}\left(W_{k+1}-W_{k}\right) .
$$

More precisely, the Riemann solver (10) is said to be consistent with the integral form of (9) if there exists a vector $S_{\Delta}\left(W_{L}, W_{R}\right)$ with

$$
\lim _{\substack{W_{L}, W_{R} \rightarrow W \\ \Delta \rightarrow 0}} S_{\Delta}\left(W_{L}, W_{R}\right)=S(W)
$$

and such that under the CFL condition (12) we have

$$
\Delta G-\Delta x S_{\Delta}\left(W_{L}, W_{R}\right)=\sum_{k=1}^{m} \sigma_{k}\left(W_{k+1}-W_{k}\right) .
$$

As it is customary, we have set $\Delta W=W_{R}-W_{L}$ and $\Delta G=G\left(W_{R}\right)-G\left(W_{L}\right)$. Observe that when the sources are not present in the model, we recover the usual definition of consistency associated with systems of conservation laws (see for instance $[18,16,8])$. 
The Godunov-type method. The Godunov-type method associated with the simple Riemann solver (10) is then defined as usual (and with classical notations) by

$$
\Delta x W_{i}^{n+1}=\int_{-\frac{\Delta x}{2}}^{0} W_{\Delta}\left(\frac{x}{\Delta t} ; W_{i}^{n}, W_{i+1}^{n}\right) d x+\int_{0}^{\frac{\Delta x}{2}} W_{\Delta}\left(\frac{x}{\Delta t} ; W_{i-1}^{n}, W_{i}^{n}\right) d x .
$$

Assuming that the approximate Riemann solver (10) is consistent with (9) and setting

$$
\mathcal{F}\left(W_{L}, W_{R}\right)=\frac{1}{2}\left\{G\left(W_{L}\right)+G\left(W_{R}\right)-\sum_{k=1}^{m}\left|\sigma_{k}\right|\left(W_{k+1}-W_{k}\right)\right\}
$$

one easily checks that under the CFL condition (12), the associated Godunovtype method reads

$$
W_{i}^{n+1}=W_{i}^{n}-\frac{\Delta t}{\Delta x}\left(\mathcal{F}_{i+\frac{1}{2}}^{n}-\mathcal{F}_{i-\frac{1}{2}}^{n}\right)+\frac{\Delta t}{2}\left(S_{i+\frac{1}{2}}^{n}+S_{i-\frac{1}{2}}^{n}\right)
$$

where for all $i \in \mathbb{Z}$

$$
\mathcal{F}_{i+\frac{1}{2}}^{n}=\mathcal{F}\left(W_{i}^{n}, W_{i+1}^{n}\right), \quad S_{i+\frac{1}{2}}^{n}=S_{\Delta}\left(W_{i}^{n}, W_{i+1}^{n}\right) .
$$

Once the approximate Riemann solver is defined according to (15), the proposed Godunov-type method is then very classical. We note however that the sources are taken into account in the definition of consistency, and then clearly receive an upwind treatment in formula (18).

\section{An asymptotic-preserving scheme for the lin- ear transport problem}

In this section, we consider the quasilinear version of (5)-(6) given by

$$
\begin{aligned}
\partial_{t} u+\partial_{x} v & =0, \\
\partial_{t} v+a^{2} \partial_{x} u & =\frac{1}{\varepsilon(t, x, u)}(f(u)-v),
\end{aligned}
$$

where $a>0$ is a given parameter satisfying the Whitham subcharacteristic condition

$$
\left|f^{\prime}(u)\right|<a \text {. }
$$

\subsection{Definition of the scheme}

We choose to define a two-state approximate Riemann solver as precised on figure 1. 


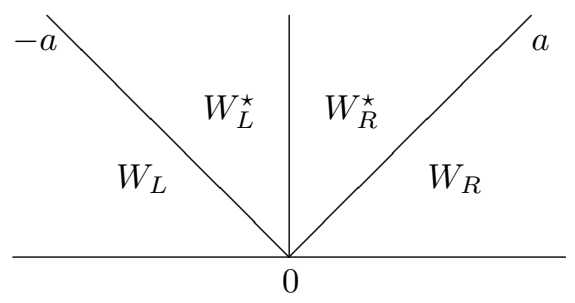

Figure 1: Illustration of the two-state approximate Riemann solver.

We propose to define the four intermediate states by imposing the following four equations. The first equation is nothing but the consistency with the integral form (15) on the first variable $u$ which writes

$$
\left(v_{R}-v_{L}\right)=-a\left(u_{L}^{\star}-u_{L}\right)+a\left(u_{R}-u_{R}^{\star}\right) .
$$

Note that the source term is not present in this equation. The last three equations are given by applying the Rankine-Hugoniot jump conditions on the $v$ variable across the three waves of the proposed approximate Riemann solver. We get

$$
\begin{aligned}
& a^{2}\left(u_{L}^{\star}-u_{L}\right)=-a\left(v_{L}^{\star}-v_{L}\right), \\
& a^{2}\left(u_{R}^{\star}-u_{L}^{\star}\right)=\frac{\Delta x}{\varepsilon} \tilde{S}\left(W_{L}, W_{R}\right), \\
& a^{2}\left(u_{R}-u_{R}^{\star}\right)=a\left(v_{R}-v_{R}^{\star}\right),
\end{aligned}
$$

where the consistent approximation $\tilde{S}\left(W_{L}, W_{R}\right)$ of $f(u)-v$ will be defined later on (to avoid cumbersome notations, $S_{\Delta}\left(W_{L}, W_{R}\right)$ in the previous section is replaced up to a factor $1 / \varepsilon$ by $\tilde{S}\left(W_{L}, W_{R}\right)$ from now on). Note that we have made the choice to take into account the source term across the stationary wave only. Importantly, we also note that the sum of these three RankineHugoniot conditions on $v$ is nothing but a consistency condition with the integral formulation (15) on $v$ :

$$
a^{2}\left(u_{R}-u_{L}\right)=-a\left(v_{L}^{\star}-v_{L}\right)+a\left(v_{R}-v_{R}^{\star}\right)+\frac{\Delta x}{\varepsilon} \tilde{S}\left(W_{L}, W_{R}\right) .
$$

Let us first remark that substracting (26) from (24) gives

$$
a\left(u_{L}^{\star}+u_{R}^{\star}\right)-a\left(u_{L}+u_{R}\right)+\left(v_{R}-v_{L}\right)=v_{R}^{\star}-v_{L}^{\star} .
$$

Using (23), we then get $v_{L}^{\star}=v_{R}^{\star}$ and by $(27)$

$$
v^{\star}:=v_{L}^{\star}=v_{R}^{\star}=\frac{v_{L}+v_{R}}{2}-\frac{a}{2}\left(u_{R}-u_{L}\right)+\frac{\Delta x}{2 a \varepsilon} \tilde{S}\left(W_{L}, W_{R}\right) .
$$

Now (23) rewrites

$$
u_{L}^{\star}+u_{R}^{\star}=u_{L}+u_{R}-\frac{v_{R}-v_{L}}{a} .
$$


This equation and (25) allow to easily find $u_{L}^{\star}$ and $u_{R}^{\star}$ by solving a linear system provided that $\tilde{S}\left(W_{L}, W_{R}\right)$ is given :

$$
\begin{aligned}
& u_{L}^{\star}=\frac{u_{L}+u_{R}}{2}-\frac{v_{R}-v_{L}}{2 a}-\frac{\Delta x}{2 a^{2} \varepsilon} \tilde{S}\left(W_{L}, W_{R}\right), \\
& u_{R}^{\star}=\frac{u_{L}+u_{R}}{2}-\frac{v_{R}-v_{L}}{2 a}+\frac{\Delta x}{2 a^{2} \varepsilon} \tilde{S}\left(W_{L}, W_{R}\right) .
\end{aligned}
$$

Equipped with these relations and thanks to (17), we can deduce the resulting numerical fluxes :

$$
\begin{aligned}
& \mathcal{F}^{u}\left(W_{L}, W_{R}\right)=\frac{1}{2}\left(v_{L}+v_{R}\right)-\frac{a}{2}\left(u_{R}-u_{L}\right)+\frac{\Delta x}{2 a \varepsilon} \tilde{S}\left(W_{L}, W_{R}\right), \\
& \mathcal{F}^{v}\left(W_{L}, W_{R}\right)=\frac{a^{2}}{2}\left(u_{L}+u_{R}\right)-\frac{a}{2}\left(v_{R}-v_{L}\right) .
\end{aligned}
$$

Under the CFL condition:

$$
\Delta t \leq \frac{\Delta x}{2 a}
$$

and using (18), the update formulas are given by:

$$
\begin{aligned}
& u_{i}^{n+1}=u_{i}^{n}-\frac{\Delta t}{\Delta x}\left(\mathcal{F}_{i+1 / 2}^{u}-\mathcal{F}_{i-1 / 2}^{u}\right), \\
& v_{i}^{n+1}=v_{i}^{n}-\frac{\Delta t}{\Delta x}\left(\mathcal{F}_{i+1 / 2}^{v}-\mathcal{F}_{i-1 / 2}^{v}\right)+\frac{\Delta t}{2 \varepsilon_{i+1 / 2}^{n}}\left(\tilde{S}_{i+1 / 2}+\tilde{S}_{i-1 / 2}\right),
\end{aligned}
$$

with $\mathcal{F}_{i+1 / 2}^{u, v}=\mathcal{F}^{u, v}\left(W_{i}^{n}, W_{i+1}^{n}\right)$ and $\tilde{S}_{i+1 / 2}=\tilde{S}\left(W_{i}^{n}, W_{i+1}^{n}\right)$.

The scheme will be complete as soon as the source term discretization $\frac{1}{\varepsilon} \tilde{S}$ is defined. We propose the following consistent formula:

$$
\begin{aligned}
\frac{1}{\varepsilon} \tilde{S}_{i+1 / 2} & =\frac{a\left(2 \psi\left(u_{i}^{n}, u_{i+1}^{n}\right)-\left(v_{i}^{n}+v_{i+1}^{n}\right)+a\left(u_{i+1}^{n}-u_{i}^{n}\right)\right)}{2 a \varepsilon_{i+1 / 2}^{n}+\Delta x}, \\
& =\frac{2 a}{2 a \varepsilon_{i+1 / 2}^{n}+\Delta x}\left(\psi\left(u_{i}^{n}, u_{i+1}^{n}\right)-v_{i+1 / 2}^{*}\right)
\end{aligned}
$$

with

and where

$$
v_{i+1 / 2}^{*}=\frac{1}{2}\left(v_{i}+v_{i+1}\right)-\frac{a}{2}\left(u_{i+1}-u_{i}\right)
$$

$$
\varepsilon_{i+1 / 2}^{n}=\varepsilon\left(t^{n}, x_{i+1 / 2}, \frac{u_{i}^{n}+u_{i+1}^{n}}{2}\right) .
$$

Here, $\psi\left(u_{i}^{n}, u_{i+1}^{n}\right)$ is any numerical flux consistent with the limit equation (3) i.e. $\psi(u, u)=f(u)$. As an example, if the HLL [18] is considered, we have:

$$
\frac{1}{\varepsilon} \tilde{S}_{i+1 / 2}=\frac{a}{2 a \varepsilon_{i+1 / 2}^{n}+\Delta x}\left(f\left(u_{i}^{n}\right)+f\left(u_{i+1}^{n}\right)-\left(v_{i}^{n}+v_{i+1}^{n}\right)\right) .
$$

The proposed definition (33) will be justified in the next section when establishing the main asymptotic and stability properties of the whole numerical scheme. 


\subsection{Main Properties}

Three results are proved in this section. First, we will show that the scheme is asymptotic-preserving with respect to both asymptotic limits $\varepsilon \rightarrow 0$ and $\varepsilon \rightarrow+\infty$. Then, we will investigate stability and maximum principle properties. Let us first consider the behavior of the scheme in the limit $\varepsilon \rightarrow \infty$. From (33), we immediately see that $\frac{1}{\varepsilon} \tilde{S}_{i+1 / 2} \rightarrow 0$ and therefore (31)-(32) becomes:

$$
\begin{aligned}
& u_{i}^{n+1}=u_{i}^{n}-\frac{\Delta t}{\Delta x}\left(\mathcal{F}_{i+1 / 2}^{u}-\mathcal{F}_{i-1 / 2}^{u}\right), \\
& v_{i}^{n+1}=v_{i}^{n}-\frac{\Delta t}{\Delta x}\left(\mathcal{F}_{i+1 / 2}^{v}-\mathcal{F}_{i-1 / 2}^{v}\right)
\end{aligned}
$$

with the flux function $\mathcal{F}_{i+1 / 2}^{v}$ is given by (29) and the flux function $\mathcal{F}_{i+1 / 2}^{u}$ writes:

$$
\mathcal{F}^{u}\left(W_{L}, W_{R}\right)=\frac{1}{2}\left(v_{L}+v_{R}\right)-\frac{a}{2}\left(u_{R}-u_{L}\right) .
$$

Finally, it is to note that our scheme simply degenerated into an HLL scheme for the linear homogeneous system issuing from (20)-(21), which is the limit system when $\varepsilon \rightarrow \infty$.

Now, we assume that $\varepsilon \rightarrow 0$ in the scheme (31)-(32)-(33). From (33) and (28), a straightforward computation gives the following limit for the flux function on $u$ :

$$
\lim _{\varepsilon \rightarrow 0} \mathcal{F}^{u}\left(W_{L}, W_{R}\right)=\psi\left(u_{L}, u_{R}\right)
$$

and therefore, the update value of $u$ in the limit $\varepsilon \rightarrow 0$ is nothing but:

$$
u_{i}^{n+1}=u_{i}^{n}-\frac{\Delta t}{\Delta x}\left(\psi_{i+1 / 2}-\psi_{i-1 / 2}\right)
$$

which is obviously consistent with the limit equation (3) according to the definition of $\psi$.

Now, inserting the limit of (33) and (29) when $\varepsilon \rightarrow 0$ into (32) yields:

$$
v_{i}^{n+1}=\left(1-2 a \frac{\Delta t}{\Delta x}\right) v_{i}^{n}+a \frac{\Delta t}{\Delta x}\left(\psi_{i+1 / 2}+\psi_{i-1 / 2}\right) .
$$

The scheme will therefore be proved to be asymptotic-preserving (i.e. consistent with $(3)$ when $\varepsilon \rightarrow 0)$ as soon as we state that $v_{i}^{n}$ is consistent with $f(u)$. This result is proved in the next two lemmae:

Lemma 1. Assume that $u_{i}^{n}$ and $v_{i}^{n}$ are given by the limit schemes (34)-(35) for small $\varepsilon$. Assume also that $u_{i}^{n}$ is the discretization of a smooth function, then, under the CFL condition (30) $\left.\Delta t=\lambda \frac{\Delta x}{2 a}, \lambda \in\right] 0,1[$ :

$$
\lim _{\Delta x \rightarrow 0, n \Delta t \text { fixed }}\left\|v_{i}^{n}-f\left(u_{i}^{n}\right)\right\|_{\infty}=0 .
$$


Proof. We first set $e_{i}^{n}:=v_{i}^{n}-f\left(u_{i}^{n}\right)$ to write from (35):

$$
\begin{aligned}
e_{i}^{n+1} & =v_{i}^{n+1}-f\left(u_{i}^{n+1}\right), \\
& =\left(1-2 a \frac{\Delta t}{\Delta x}\right) v_{i}^{n}+a \frac{\Delta t}{\Delta x}\left(\psi_{i+1 / 2}+\psi_{i-1 / 2}\right)-f\left(u_{i}^{n+1}\right) .
\end{aligned}
$$

Using the consistency of $\psi$ and a Taylor expansion of $f\left(u_{i}^{n+1}\right)$ we get:

$$
\begin{aligned}
e_{i}^{n+1} & =\left(1-2 a \frac{\Delta t}{\Delta x}\right) v_{i}^{n}+a \frac{\Delta t}{\Delta x}\left(2 f\left(u_{i}^{n}\right)+O(\Delta x)\right)-f\left(u_{i}^{n}\right)+O(\Delta x) \\
& =\left(1-2 a \frac{\Delta t}{\Delta x}\right) e_{i}^{n}+O(\Delta x) \\
& =\cdots=\left(1-2 a \frac{\Delta t}{\Delta x}\right)^{n+1} e_{i}^{0}+O(\Delta x) \sum_{k=0}^{n}\left(1-2 a \frac{\Delta t}{\Delta x}\right)^{k} \\
& =\left(1-2 a \frac{\Delta t}{\Delta x}\right)^{n+1} e_{i}^{0}+\frac{1-\left(1-2 a \frac{\Delta t}{\Delta x}\right)^{n+1}}{1-\left(1-2 a \frac{\Delta t}{\Delta x}\right)} O(\Delta x),
\end{aligned}
$$

and since, due to the CFL condition (30) $\left|1-2 a \frac{\Delta t}{\Delta x}\right|<1$, we obtain $\lim _{\Delta x \rightarrow 0, n \Delta t \text { fixed }}\left\|e_{i}^{n}\right\|_{\infty}=$ 0 which is the expected result.

Lemma 2. Assume that $f$ is locally Lipschitz continuous and that $\psi$ is a TVD flux and satisfies:

$$
\exists \alpha>0, \quad \sum_{i \in \mathbb{Z}}\left|\psi_{i+1 / 2}-f\left(u_{i}^{n}\right)\right| \leq \alpha T V\left(u^{n}\right),
$$

where TV $\left(u^{n}\right)$ denotes the total variation of $u^{n}$. Then, under the CFL condition (30):

$$
\forall n \in \mathbb{N}, \quad \lim _{\Delta x \rightarrow 0}\left\|v^{n}-f\left(u^{n}\right)\right\|_{1}=0 .
$$

Proof. As previously, we set $e_{i}^{n}:=v_{i}^{n}-f\left(u_{i}^{n}\right)$ to write from (35):

$$
\begin{aligned}
e_{i}^{n+1} & =\left(1-2 a \frac{\Delta t}{\Delta x}\right) v_{i}^{n}+a \frac{\Delta t}{\Delta x}\left(\psi_{i+1 / 2}+\psi_{i-1 / 2}\right)-f\left(u_{i}^{n+1}\right), \\
& =\left(1-2 a \frac{\Delta t}{\Delta x}\right) e_{i}^{n}+a \frac{\Delta t}{\Delta x}\left(\psi_{i+1 / 2}+\psi_{i-1 / 2}-2 f\left(u_{i}^{n}\right)\right)-\left(f\left(u_{i}^{n+1}\right)-f\left(u_{i}^{n}\right)\right),
\end{aligned}
$$

to get:

$$
\left|e_{i}^{n+1}\right| \leq\left(1-2 a \frac{\Delta t}{\Delta x}\right)\left|e_{i}^{n}\right|+a \frac{\Delta t}{\Delta x}\left|\psi_{i+1 / 2}+\psi_{i-1 / 2}-2 f\left(u_{i}^{n}\right)\right|+\left|f\left(u_{i}^{n+1}\right)-f\left(u_{i}^{n}\right)\right|,
$$

multiplying by $\Delta x$ and summing over $i \in \mathbb{Z}$ we have:

$$
\begin{aligned}
\left\|e^{n+1}\right\|_{1} & \leq\left(1-2 a \frac{\Delta t}{\Delta x}\right)\left\|e_{i}^{n}\right\|_{1} \\
& +a \frac{\Delta t}{\Delta x} \sum_{i \in \mathbb{Z}} \Delta x\left|\psi_{i+1 / 2}+\psi_{i-1 / 2}-2 f\left(u_{i}^{n}\right)\right|+\sum_{i \in \mathbb{Z}} \Delta x\left|f\left(u_{i}^{n+1}\right)-f\left(u_{i}^{n}\right)\right| .
\end{aligned}
$$


Using the condition (36), we obtain:

$$
\sum_{i \in \mathbb{Z}}\left|\psi_{i+1 / 2}+\psi_{i-1 / 2}-2 f\left(u_{i}^{n}\right)\right| \leq(2 \alpha+L) \operatorname{TV}\left(u^{n}\right),
$$

and since $f$ is locally Lipschitz continuous, we can write:

$$
\begin{aligned}
\sum_{i \in \mathbb{Z}}\left|f\left(u_{i}^{n+1}\right)-f\left(u_{i}^{n}\right)\right| & \leq L \sum_{i \in \mathbb{Z}}\left|u_{i}^{n+1}-u_{i}^{n}\right| \\
& \leq L \frac{\Delta t}{\Delta x} \sum_{i \in \mathbb{Z}}\left|\psi_{i+1 / 2}-\psi_{i-1 / 2}\right|, \\
& \leq 2 L \alpha \frac{\Delta t}{\Delta x} \operatorname{TV}\left(u^{n}\right) .
\end{aligned}
$$

Finally:

$$
\left\|e^{n+1}\right\|_{1} \leq\left(1-2 a \frac{\Delta t}{\Delta x}\right)\left\|e^{n}\right\|_{1}+a \Delta t\left(2 \alpha+1+2 L \frac{\alpha}{a}\right) \operatorname{TV}\left(u^{n}\right),
$$

and since $\psi$ is a TVD flux, $\operatorname{TV}\left(u^{n}\right)<\infty$, therefore:

$$
\left\|e^{n+1}\right\|_{1} \leq\left(1-2 a \frac{\Delta t}{\Delta x}\right)^{n+1}\left\|e^{0}\right\|_{1}+\frac{1-\left(1-2 a \frac{\Delta t}{\Delta x}\right)^{n+1}}{1-\left(1-2 a \frac{\Delta t}{\Delta x}\right)} O(\Delta x),
$$

hence we have the expected limit under the CFL condition (30).

It is to note that the assumption (36) is not restrictive. Indeed, most schemes satisfy it, including the classical schemes: HLL, Lax-Friedrichs, Murmann-Roe, Lax-Wendroff, Beam-Warming... (see [16, 21, 24]). In addition, the scheme possesses the usual robustness and stability properties in both limit regimes.

Lemma 3. As soon as the CFL condition (30) is satisfied then:

1. The scheme preserves the maximum principle, the entropy inequalities and is TVD in the diagonal variables $v \pm$ au in the limit of $\varepsilon \rightarrow \infty$.

2. The scheme preserves all the properties satisfied by the choice of $\psi$ in the limit of $\varepsilon \rightarrow 0$.

Proof. The proof is straightforward since the scheme degenerates into the HLL scheme in the linear transport regime and into the chosen scheme (34) in the relaxation limit.

To conclude this section, let us underline that our scheme conserves the stability properties inherited from the HLL scheme and the choice of $\psi$ :

Lemma 4. Let us assume that the CFL condition (30) holds and that the scheme (34) is $L^{2}$-stable under this CFL condition. Then, for every fixed $\varepsilon>0$, the scheme (31)-(32)-(33) is also $L^{2}$-stable. 
Proof. Introducing $\mu=\frac{\Delta x}{2 a \varepsilon+\Delta x} \in[0,1]$ and $U=(u, v)^{\top}$, the scheme (31)(32)-(33) can be rewritten as:

$$
\begin{aligned}
U_{i}^{n+1} & =(1-\mu)\left(U_{i}^{n}-\frac{\Delta t}{\Delta x}\left(\mathcal{F}_{i+1 / 2}-\mathcal{F}_{i-1 / 2}\right)\right) \\
& +\mu\left(\begin{array}{c}
u_{i}^{n}-\frac{\Delta t}{\Delta x}\left(\psi_{i+1 / 2}-\psi_{i-1 / 2}\right) \\
v_{i}^{n}\left(1-2 a \frac{\Delta t}{\Delta x}\right)+a \frac{\Delta t}{\Delta x}\left(\psi_{i+1 / 2}+\psi_{i-1 / 2}\right)
\end{array}\right),
\end{aligned}
$$

where $\mathcal{F}$ is the HLL flux for the linear transport regime and $\psi_{i+1 / 2}=\psi\left(u_{i}^{n}, u_{i+1}^{n}\right)$. Therefore, the scheme is nothing but a convex combination between two other schemes. Since the HLL scheme is $L^{2}$-stable under the CFL condition (30), the stability will be established as soon as the following scheme is $L^{2}$-stable:

$$
U_{i}^{n+1}=\left(\begin{array}{c}
u_{i}^{n}-\frac{\Delta t}{\Delta x}\left(\psi_{i+1 / 2}-\psi_{i-1 / 2}\right) \\
v_{i}^{n}\left(1-2 a \frac{\Delta t}{\Delta x}\right)+a \frac{\Delta t}{\Delta x}\left(\psi_{i+1 / 2}+\psi_{i-1 / 2}\right)
\end{array}\right),
$$

which amplification matrix is given by:

$$
A(\xi)=\left(\begin{array}{cc}
\alpha(\xi) & 0 \\
\beta(\xi) & 1-2 a \frac{\Delta t}{d x}
\end{array}\right),
$$

where $\alpha(\xi)$ is the amplification coefficient associated to the scheme (34) and $\beta(\xi)$ is a given function. The eigenvalues of $A(\xi)$ are obviously $\alpha(\xi)$ and $1-2 a \frac{\Delta t}{d x}$. Under the CFL condition (30), $\left|1-2 a \frac{\Delta t}{d x}\right|<1$ and since (34) is also assumed to be $L^{2}$-stable under the same CFL condition, $|\alpha(\xi)|<1$. The scheme (37) is hence also $L^{2}$-stable.

To conclude this paragraph, let us point out that, since the hypotheses stated in [12] are satisfied, it may be interesting to investigate the first-order corrector in $\varepsilon$. A straightforward computation on the scheme (31)-(32)-(33) in the limit $\varepsilon \rightarrow 0$ (with a fixed $\Delta x$ ) gives:

$$
\begin{aligned}
u_{i}^{n+1} & =u_{i}^{n}-\frac{\Delta t}{\Delta x}\left(\psi_{i+1 / 2}-\psi_{i-1 / 2}\right) \\
& +\varepsilon \frac{2 a \Delta t}{\Delta x^{2}}\left(\psi_{i+1 / 2}-\psi_{i-1 / 2}-\frac{v_{i+1}^{n}-v_{i-1}^{n}}{2}+\frac{a\left(u_{i+1}^{n}-2 u_{i}^{n}+u_{i-1}^{n}\right)}{2}\right)+\mathcal{O}\left(\varepsilon^{2}\right) .
\end{aligned}
$$

Interestingly, it is therefore possible to choose $\psi$ in order to recover a discrete form of the first-order corrected equation of Chen-Levermore and Liu:

$$
\partial_{t} u+\partial_{x} f(u)=\varepsilon \partial_{x}\left(\left(a^{2}-f^{\prime}(u)^{2}\right) \partial_{x} u\right) .
$$

One corresponding choice of $\psi$ is:

$$
\psi_{i+1 / 2}=\frac{f\left(u_{i+1}^{n}\right)+f\left(u_{i}^{n}\right)}{2}-f^{\prime}\left(\frac{u_{i}^{n}+u_{i+1}^{n}}{2}\right)\left(u_{i+1}^{n}-u_{i}^{n}\right) .
$$




\section{An asymptotic-preserving scheme for the non- linear transport problem with relaxation-type source term}

\subsection{Definition of the scheme}

Now we propose to extend the scheme developed in the previous section to the nonlinear case (5)-(6). As before, we consider a two-state approximate Riemann solver as illustrated on figure (1). Here, $a$ represents a positive constant greater than the wave speeds of the system. In order to characterize the intermediate states, we follow the same lines as in the linear case and we first impose the consistency with the integral formulation on $u$, which writes

$$
v_{R}-v_{L}=-a\left(u_{L}^{\star}-u_{L}\right)+a\left(u_{R}-u_{R}^{\star}\right),
$$

and then the Rankine-Hugoniot conditions across the three waves on the $v$ component :

$$
\begin{aligned}
g_{L}^{\star}-g_{L} & =-a\left(v_{L}^{\star}-v_{L}\right), \\
g_{R}^{\star}-g_{L}^{\star} & =\frac{\Delta x}{\varepsilon} \tilde{S}\left(W_{L}, W_{R}\right), \\
g_{R}-g_{R}^{\star} & =a\left(v_{R}-v_{R}^{\star}\right) .
\end{aligned}
$$

These equations are non linear and cannot be solved explicitly if $g_{L R}^{\star}$ are defined by $g\left(u_{L R}^{\star}, v_{L R}^{\star}\right)$. For the sake of linearity, we choose to consider $g_{L R}^{\star}$ as additional unknowns instead. In order to close the system, we therefore need to add two new relations.

As in the linear case, we first propose to impose the continuity of $v$ :

$$
v_{L}^{\star}=v_{R}^{\star}
$$

Then we propose the following linearization of $g$ :

$$
g_{R}^{\star}-g_{L}^{\star}=K\left(u_{R}^{\star}-u_{L}^{\star}\right),
$$

where $K$ is a Lipschitz constant of $g$. These six relations easily allow us to fully determine the intermediate states. Hence, we can compute the form of the numerical fluxes and we get

$$
\begin{aligned}
& \mathcal{F}^{u}\left(W_{L}, W_{R}\right)=\frac{1}{2}\left(v_{L}+v_{R}\right)-\frac{a}{2}\left(u_{R}-u_{L}\right)+\frac{a \Delta x}{2 K \varepsilon} \tilde{S}\left(W_{L}, W_{R}\right), \\
& \mathcal{F}^{v}\left(W_{L}, W_{R}\right)=\frac{1}{2}\left(g_{L}+g_{R}\right)-\frac{a}{2}\left(v_{R}-v_{L}\right) .
\end{aligned}
$$


As previously, we thus create a finite volumes scheme which will be completed, under the CFL condition (30), as soon as a consistent approximation of the source term is set for $\tilde{S}$. We propose the following form:

$$
\begin{aligned}
\frac{1}{\varepsilon} \tilde{S}_{i+1 / 2} & =\frac{K\left(2 \psi\left(u_{i}^{n}, u_{i+1}^{n}\right)-\left(v_{i}^{n}+v_{i+1}^{n}\right)+a\left(u_{i+1}^{n}-u_{i}^{n}\right)\right)}{2 K \varepsilon_{i+1 / 2}^{n}+a \Delta x} \\
& =\frac{2 K}{2 K \varepsilon_{i+1 / 2}^{n}+a \Delta x}\left(\psi\left(u_{i}^{n}, u_{i+1}^{n}\right)-v_{i+1 / 2}^{*}\right)
\end{aligned}
$$

where $\psi$ is once again any numerical flux consistent with the limit equation (3). Finally, the updates are still given by (31)-(32). Let us emphasize that this scheme is indeed an extension of the one defined in the previous section since in the linear case $K=a^{2}$ and (44)-(45)-(46) coincide with (28)-(29)-(33).

\subsection{Main properties}

In this section, we investigate the behavior of the scheme for the nonlinear system (5)-(6).

In the limit of $\varepsilon \rightarrow \infty$, we immediately see that the scheme degenerates into the HLL scheme.

In the limit of $\varepsilon \rightarrow 0$, the update of $u$ is once again nothing but (34) which is obviously consistent with the relaxation limit (3). To achieve the asymptoticpreserving property, we have the following result:

Lemma 5. Let us assume that the hypothesis of lemma 2 holds. Let us denote $K$ and $K^{\prime}$ the Lipschitz constants of $g$ w.r.t. $u$ and $v$ respectively. Then, provided that $2 a^{2} \geq 2 K \geq a K^{\prime}$ and under the CFL condition:

$$
a \frac{\Delta t}{\Delta x} \leq \frac{1}{2} \min \left(1, \frac{a^{2}}{2 K-a K^{\prime}}, \frac{4 a^{2}}{a^{2}+3 K}\right),
$$

we have:

$$
\lim _{\Delta x \rightarrow 0, n \Delta t \text { fixed }}\left\|v_{i}^{n}-f\left(u_{i}^{n}\right)\right\|_{1}=0 .
$$

Furthermore, if $u_{i}^{n}$ is a discretization of a smooth function, then:

$$
\lim _{\Delta x \rightarrow 0, n \Delta t \text { fixed }}\left\|v_{i}^{n}-f\left(u_{i}^{n}\right)\right\|_{\infty}=0 .
$$

Remark. For stability considerations, recall that the parameter a has to be taken greater than the largest eigenvalue of the system (1)-(2). For instance:

$$
K^{\prime}+\sqrt{K^{\prime 2}+4 K} \leq 2 a,
$$

which can always be fulfilled together with the assumption $2 a^{2} \geq 2 K \geq a K^{\prime}$. Moreover, when $g$ allows to choose $2 K=a K^{\prime}$, the usual CFL condition (30) is recovered. 
Proof. As in the linear case, we set $e_{i}^{n}:=v_{i}^{n}-f\left(u_{i}^{n}\right)$. We first establish that $v \in B V$. Starting from (32) with (45)-(46) and involving the hypothesis (36), we get:

$$
\begin{aligned}
v_{i}^{n+1} & =\left(1-a \frac{\Delta t}{\Delta x}-\frac{K \Delta t}{a \Delta x}\right) v_{i}^{n}+\left(\frac{a \Delta t}{2 \Delta x}-\frac{K \Delta t}{2 a \Delta x}\right)\left(v_{i+1}^{n}+v_{i-1}^{n}\right) \\
& -\frac{\Delta t}{2 \Delta x}\left(g_{i+1}-g_{i-1}\right)+\frac{K \Delta t}{2 \Delta x}\left(u_{i+1}^{n}-u_{i-1}^{n}\right)+\frac{K \Delta t}{a \Delta x}\left(\psi_{i+1 / 2}+\psi_{i-1 / 2}\right),
\end{aligned}
$$

so that:

$$
\begin{aligned}
T V\left(v^{n+1}\right) & =\sum_{i \in \mathbb{Z}}\left|v_{i+1}^{n+1}-v_{i}^{n+1}\right|, \\
& \leq\left(1-2 K \frac{\Delta t}{a \Delta x}\right) T V\left(v^{n}\right)+C \frac{\Delta t}{\Delta x} T V\left(u^{n}\right)+\frac{\Delta t}{\Delta x} \sum_{i \in \mathbb{Z}}\left|g_{i+1}-g_{i}\right|,
\end{aligned}
$$

where $C=C\left(K, K^{\prime}, a\right)$ is a positive constant. Now, using the fact that $g$ is locally Lipschitz continuous, we end up with:

$$
\begin{aligned}
T V\left(v^{n+1}\right) & \leq\left(1+\left(K^{\prime}-\frac{2 K}{a}\right) \frac{\Delta t}{\Delta x}\right) T V\left(v^{n}\right)+(C+K) T V\left(u^{n}\right), \\
& \leq\left(1+\left(K^{\prime}-\frac{2 K}{a}\right) \frac{\Delta t}{\Delta x}\right)^{n} T V\left(v^{0}\right) \\
& +(C+K) T V\left(u^{n}\right) \frac{1-\left(1+\left(K^{\prime}-\frac{2 K}{a}\right) \frac{\Delta t}{\Delta x}\right)^{n+1}}{1-\left(1+\left(K^{\prime}-\frac{2 K}{a}\right) \frac{\Delta t}{\Delta x}\right)},
\end{aligned}
$$

and since the choice of $\psi$ implies that $u \in B V$, then $v \in B V$ as soon as $1+\left(K^{\prime}-\frac{2 K}{a}\right) \frac{\Delta t}{\Delta x} \leq 1$. This last condition is a consequence of the CFL condition (47) and the hypothesis $2 K \geq a K^{\prime}$. Then we have:

$$
\begin{aligned}
e_{i}^{n+1} & =v_{i}^{n+1}-f\left(u_{i}^{n+1}\right), \\
& =\left(1-\frac{a \Delta t}{\Delta x}-\frac{K \Delta t}{a \Delta x}\right) e_{i}^{n}+\left(\frac{a \Delta t}{2 \Delta x}-\frac{K \Delta t}{2 a \Delta x}\right)\left(e_{i+1}^{n}+e_{i-1}^{n}\right) \\
& +\left(\frac{a \Delta t}{2 \Delta x}-\frac{K \Delta t}{2 a \Delta x}\right)\left(f\left(u_{i+1}^{n}\right)-f\left(u_{i}^{n}\right)+f\left(u_{i-1}^{n}\right)-f\left(u_{i}^{n}\right)\right) \\
& +f\left(u_{i}^{n}\right)-f\left(u_{i}^{n+1}\right)+\frac{K \Delta t}{a \Delta x}\left(\psi_{i+1 / 2}-f\left(u_{i}^{n}\right)+\psi_{i-1 / 2}-f\left(u_{i-1}^{n}\right)\right) \\
& -\frac{\Delta t}{2 \Delta x}\left(g_{i+1}-g_{i-1}\right)+\frac{K \Delta t}{2 \Delta x}\left(u_{i+1}^{n}-u_{i-1}^{n}\right)+\frac{K \Delta t}{a \Delta x}\left(f\left(u_{i-1}^{n}\right)-f\left(u_{i}^{n}\right)\right) .
\end{aligned}
$$

Involving the fact that $f$ and $g$ are locally Lipschitz continuous, $2 a^{2} \geq 2 K \geq a K^{\prime}$ and the property (36), we get:

$$
\left\|e^{n+1}\right\|_{1} \leq\left(1-\left(a+\frac{3 K}{a}\right) \frac{\Delta t}{2 \Delta x}\right)\left\|e^{n}\right\|_{1}+C \Delta x\left(T V\left(u^{n}\right)+T V\left(v^{n}\right)\right),
$$


which once again leads to the expected result.

Similarly, starting from (48) and using Taylor expansions whenever the functions are smooth, we have:

$e_{i}^{n+1}=\left(1-\left(a+\frac{3 K}{a}\right) \frac{\Delta t}{2 \Delta x}\right) e_{i}^{n}+\frac{K \Delta t}{a \Delta x}\left(\psi_{i+1 / 2}-f\left(u_{i}^{n}\right)+\psi_{i-1 / 2}-f\left(u_{i-1}^{n}\right)\right)+O(\Delta x)$,

and involving (36), we obtain:

$e_{i}^{n+1}=\left(1-\left(a+\frac{3 K}{a}\right) \frac{\Delta t}{2 \Delta x}\right) e_{i}^{n}+O(\Delta x)$

which gives the expected result.

In addition, the scheme still possesses the usual robustness and stability properties in both limit regimes.

Lemma 6. As soon as the CFL condition (30) is satisfied then:

1. The scheme preserves the maximum principle, the entropy inequalities and is TVD in the diagonal variables $v \pm$ au in the limit of $\varepsilon \rightarrow \infty$.

2. The scheme preserves all the properties satisfied by the choice of $\psi$ in the limit of $\varepsilon \rightarrow 0$.

3. The scheme is $L^{2}$ stable as soon as the scheme (34) is so.

Proof. The proof is straightforward since the scheme still degenerates into the HLL scheme in the linear transport regime and into the chosen scheme (34) in the relaxation limit. Moreover, the proof of the stability result is exactly the same that in the linear case.

\section{An asymptotic-preserving scheme for the non- linear transport problem with general source term}

We finally design a scheme for the case of general source term (1)-(2). Compared to the previous scheme, which was based on a relaxation-type source term, we just have to correctly take into account the effect of a generic source term.

Therefore, the updates will still be governed by the scheme (31)-(32) using the numerical fluxes (44)-(45). The scheme will therefore be complete as soon as a relevant discrete definition of the source term is given.

Let us emphasize that there is an additional difficulty since the expression of the flux function $f$ is generally only implicitly defined inside $S$. As opposed to the relaxation model studied in the previous sections, the equilibrium map 
is not explicitly given. Our main goal is to design a discrete version of $S$ that possess the same behavior in the limit $\varepsilon \rightarrow 0$ that the previous scheme:

$$
\lim _{\varepsilon \rightarrow 0, \Delta x \text { fixed }} \frac{1}{\varepsilon} \tilde{S}_{i+1 / 2}=\frac{2 K}{a \Delta x}\left(\psi_{i+1 / 2}-v_{i+1 / 2}^{\star}\right) .
$$

Indeed, if such a behavior is satisfied, then the control of the numerical flux $\psi$ is still possible.

On the other hand, the discretization of the source term is expected to be consistent:

$$
\lim _{\Delta x \rightarrow 0, \varepsilon \text { fixed }} \frac{1}{\varepsilon} \tilde{S}_{i+1 / 2}=\frac{S\left(u_{i}, v_{i}\right)+S\left(u_{i+1}, v_{i+1}\right)}{2 \varepsilon_{i+1 / 2}} .
$$

Among the admissible choices, we propose to consider the following source-term discretization:

$$
\begin{aligned}
\frac{1}{\varepsilon} \tilde{S}_{i+1 / 2} & =\frac{K \Delta x\left(2 \psi\left(u_{i}^{n}, u_{i+1}^{n}\right)-\left(v_{i}^{n}+v_{i+1}^{n}\right)+a\left(u_{i+1}^{n}-u_{i}^{n}\right)\right)}{2 K \varepsilon_{i+1 / 2}^{n}+a \Delta x^{2}} \\
& +\frac{\left(\varepsilon_{i+1 / 2}^{n}\right)^{\delta}}{2\left(\varepsilon_{i+1 / 2}^{n}\right)^{1+\delta}+\Delta x}\left(S\left(u_{i+1}^{n}, v_{i+1}^{n}\right)+S\left(u_{i}^{n}, v_{i}^{n}\right)\right),
\end{aligned}
$$

where $\delta>0$ is a given parameter. We immediately point out that this definition of $\tilde{S}$ obviously satisfies the two required properties.

This choice of $\tilde{S}$ is designed to be relevant in both asymptotic regimes. Indeed, it allows to recover the behavior of the previous scheme in these two limit regimes. Since the limit schemes are still respectively given by the HLL scheme in the transport limit and (34) in the relaxation regime, this new choice of $\tilde{S}$ preserves the results of lemmae 5 and 6 .

To end this section, we emphasize the fact that the parameter $\delta$ is free in the definition of $\tilde{S}$. However, numerical experiments show that too large values of $\delta$ decrease the efficiency of the scheme in transitional regimes.

\section{$6 \quad$ Numerical experiments}

In this section, we illustrate the relevance of the scheme on several test-cases. The splitting procedure we use when dealing with (5)-(6) is as follows. In a first step, we exactly integrate the relaxation part:

$$
\begin{aligned}
& u_{i}^{n+1,-}=u_{i}^{n}, \\
& v_{i}^{n+1,-}=f\left(u_{i}^{n}\right)+\left(v_{i}^{n}-f\left(u_{i}^{n}\right)\right) e^{-\frac{\Delta t}{\varepsilon}},
\end{aligned}
$$

when $\varepsilon$ is a constant. Otherwise, the exact integration is not possible anymore. In such cases, a simple rectangular rule will be used in numerical experiments. The second step simply consists in using the HLL scheme for the transport part (7)-(8). 


\subsection{Steady solution to the linear system}

We first consider the steady solutions of the linear system (20)-(21) when $\varepsilon$ is a constant and $f(u)=a u$ :

$$
\begin{aligned}
\partial_{x} v & =0, \\
a^{2} \partial_{x} u & =\frac{1}{\varepsilon}(a u-v) .
\end{aligned}
$$

The solution is straightforwardly:

$$
\begin{aligned}
& v=v_{0}, \\
& u=\frac{v_{0}}{a}+\left(u_{0}-\frac{v_{0}}{a}\right) e^{\frac{x}{a \varepsilon}} .
\end{aligned}
$$

where $u_{0}$ represents the value of $u$ at point $x=0$.

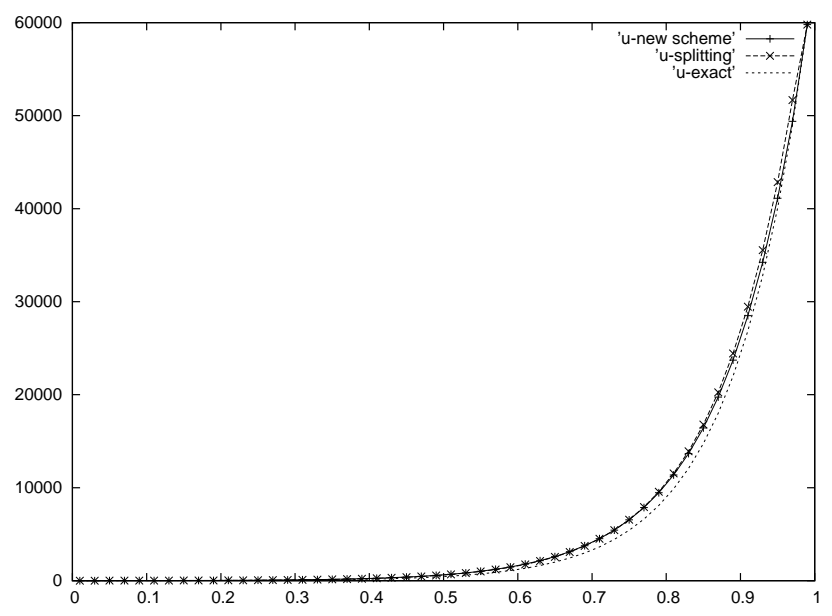

Figure 2: Steady-state test-case: exact and approximated solution at $t=0.5$.

Figure 2 shows the comparison between the exact and computed solutions at time $t=0.5$ for $a=1, v_{0}=1, u_{0}=4, \varepsilon=1$ and $\Delta x=0.01$. Initial and boundary conditions are fixed to the exact solution. We can remark a very good agreement between the exact solution and the approximations.

\subsection{Unsteady linear test-case}

Now we consider the solutions of (20)-(21) with $f(u)=\frac{u^{2}}{2}$. In all tests, $a$ is fixed to 2 . The objective of this test-case is to check the reliability of our 
scheme in every regime. To do so, we perform three different simulations with $g(u, v)=a^{2} u$ and involving three different values of $\varepsilon: \varepsilon=10$-transport regime governed by (7)-(8)-, $\varepsilon=0.03$-intermediate regime- and $\varepsilon=10^{-4}$-relaxation regime here governed by Burgers' equation.

The initial data is given by:

$$
\begin{aligned}
& u(0, x)=\left\{\begin{array}{l}
1 \text { if }\left|x-\frac{1}{2}\right|<0.1, \\
0 \text { elsewhere }
\end{array}\right. \\
& v(0, x)=f(u(0, x))
\end{aligned}
$$

We systematically compare the solutions given on a uniform mesh with $\Delta x=$ 0.05 at time $t=0.1$ by our scheme with both the splitting scheme and a reference solution. We have adopted two different forms of the relaxed flux function $\psi$ using the HLL and Lax-Wendroff methods. The reference solution is obtained by converging the splitting method. This reference solution coincides with the exact solution in both asymptotic regimes.
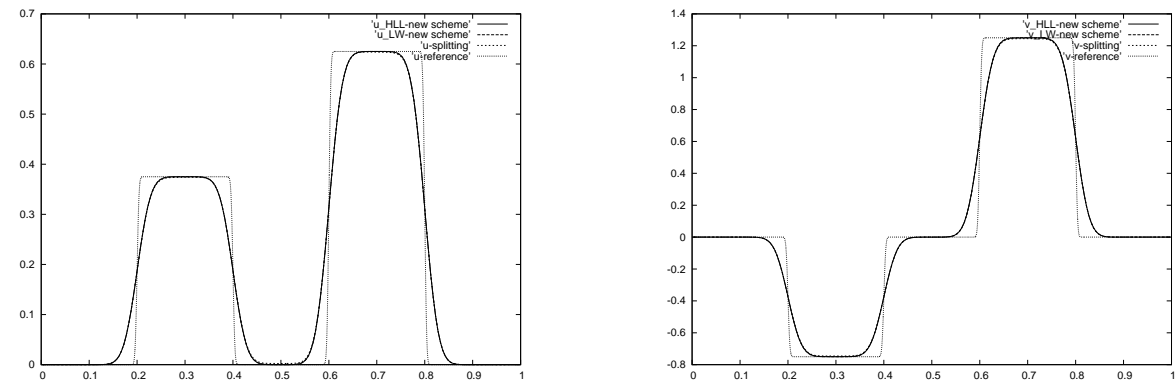

Figure 3: Linear test-case: transport regime $\varepsilon=10$. (l) $u,(\mathrm{r}) v$.

As expected, we see a good agreement between the reference and approximate solution in both asymptotic regimes (figures 3 and 5). Let us remark that the transitional regimes are also well captured as shown in figure 4.

As expected, the Lax-Wendroff and HLL fluxes have very small differences on the result in the transport and intermediate regimes. Of course, we recover the expected behavior of these schemes in the relaxation regime.

Figure 5 also shows the error $|v-f(u)|$. This result illustrates lemmae 1 and 2: in zones where $u$ is smooth, $|v-f(u)|$ converges pointwise to 0 and the $L^{1}$ norm also converges. The convergence is here of order 2 for the Lax-Wendroff 

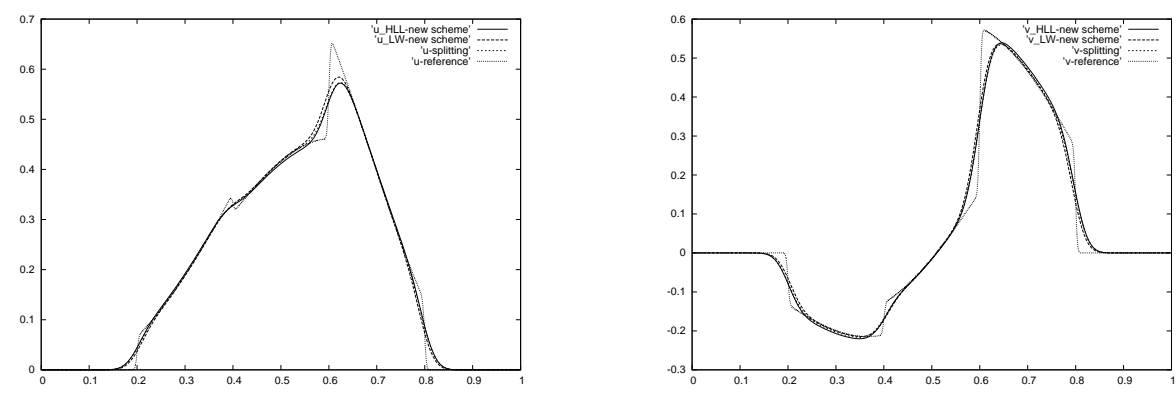

Figure 4: Linear test-case: intermediate regime $\varepsilon=0.03$. (l) $u$, (r) $v$.
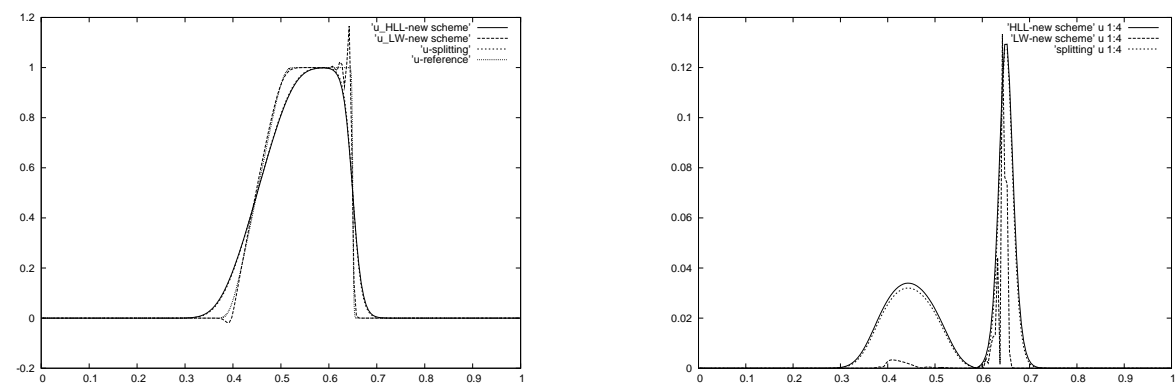

Figure 5: Linear test-case: relaxation regime (Burgers) $\varepsilon=10^{-4}$. (l) $u,(\mathrm{r})$ $|v-f(u)|$. 
flux. Let us point nonetheless that in the shock, $|v-f(u)|$ does not converge pointwise. At last, one clearly sees on this test-case involving a small value of $\varepsilon$ the interest of being able to choose the flux function $\psi$ in order to approximate the nonlinear limit system (3).

\subsection{Fully nonlinear test-cases}

Here we test the behavior of the scheme in the general case (1)-(2). Since the source-term depends on $t, u$ and $v$, the exact integration for the splitting technique (50) is not relevant anymore. The basic strategy to extend the splitting scheme in such a framework is to use a quadrature rule to integrate the source term. In order to have an explicit scheme, a basic rectangular rule is a straightforward choice. We will perform computations in a case where an exact solution can be found to assess the relevance of our scheme. The computations are performed using two different numerical fluxes to approximate the limit regime (3) i.e. in equation (49), $\psi$ will be either the HLL or the Lax-Wendroff flux.

This test case is based on:

$$
\begin{aligned}
g(u, v) & =\frac{u^{2}}{2}+2 u+v, \\
S(u, v) & =u v+u+v, \\
\varepsilon & =1,
\end{aligned}
$$

equipped with the corresponding IC and $\mathrm{BC}$, the exact solution is given by:

$$
\begin{aligned}
& u(t, x)=e^{-t} \cosh (x), \\
& v(t, x)=e^{-t} \sinh (x),
\end{aligned}
$$

finally:

$$
f(u)=\frac{-u}{1+u} .
$$

Figure 6 shows the results obtained with our scheme using 64 points compared with the exact solution. Once again, these results are in good agreement with our scheme, even on this very stiff problem. This behavior is confirmed on table 1 , which summarizes the $L^{2}$-errors on $u$ and $v$. We note that in this configuration where $\varepsilon$ is large the impact of the choice of $\psi$ is neglectable.

\subsection{Variable $\varepsilon$ test-cases}

These test-cases deal with configurations involving discontinuous $\varepsilon$ from large to small values. They are proposed by Jin et al. in [19] where a domain decomposition technique is derived for such problems. 

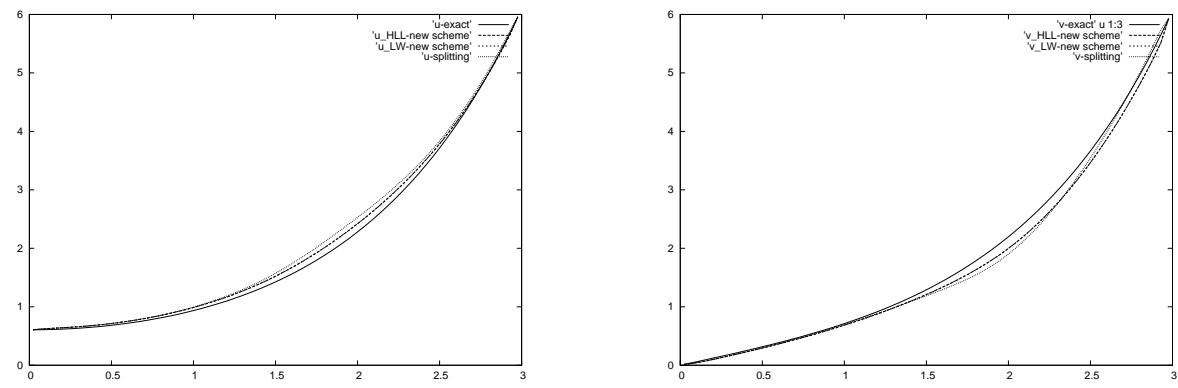

Figure 6: NL TC1: (l) $u,(\mathrm{r}) v$ at time $t=0.5$. Exact solution compared with standard splitting on 64 points and scheme (1)-(2).

\begin{tabular}{l||c|c||c|c||c|c} 
\# of pts & $\begin{array}{c}L^{2} \text { err on } u \\
\text { HLL }\end{array}$ & $\begin{array}{c}L^{2} \text { err on } v \\
\text { HLL }\end{array}$ & $\begin{array}{c}L^{2} \text { err on } u \\
\text { LW }\end{array}$ & $\begin{array}{c}L^{2} \text { err on } v \\
\text { LW }\end{array}$ & $\begin{array}{c}L^{2} \text { err on } u \\
\text { Splitting }\end{array}$ & $\begin{array}{c}L^{2} \text { err on } v \\
\text { Splitting }\end{array}$ \\
\hline 64 & $2.00 \mathrm{e}-2$ & $5.70 \mathrm{e}-2$ & $1.20 \mathrm{e}-2$ & $4.77 \mathrm{e}-2$ & $5.12 \mathrm{e}-2$ & $5.68 \mathrm{e}-2$ \\
128 & $5.91 \mathrm{e}-3$ & $1.91 \mathrm{e}-2$ & $5.75 \mathrm{e}-3$ & $1.76 \mathrm{e}-2$ & $1.48 \mathrm{e}-2$ & $1.76 \mathrm{e}-2$ \\
256 & $1.61 \mathrm{e}-3$ & $5.52 \mathrm{e}-3$ & $1.58 \mathrm{e}-3$ & $5.31 \mathrm{e}-3$ & $3.99 \mathrm{e}-3$ & $4.94 \mathrm{e}-3$ \\
512 & $4.20 \mathrm{e}-4$ & $1.48 \mathrm{e}-3$ & $4.17 \mathrm{e}-4$ & $1.46 \mathrm{e}-3$ & $1.04 \mathrm{e}-3$ & $1.32 \mathrm{e}-3$ \\
1024 & $1.07 \mathrm{e}-4$ & $3.85 \mathrm{e}-4$ & $1.07 \mathrm{e}-4$ & $3.81 \mathrm{e}-4$ & $2.66 \mathrm{e}-4$ & $3.40 \mathrm{e}-4$ \\
2048 & $2.72 \mathrm{e}-5$ & $9.80 \mathrm{e}-5$ & $2.71 \mathrm{e}-5$ & $9.75 \mathrm{e}-5$ & $6.75 \mathrm{e}-5$ & $8.66 \mathrm{e}-5$
\end{tabular}

Table 1: NL TC: $L^{2}$-errors. 
For all of these cases, $g(u, v)=u, \varepsilon$ is given by:

$$
\varepsilon=\left\{\begin{array}{l}
1 \text { if } x<1, \\
2.10^{-3} \text { otherwise. }
\end{array}\right.
$$

Here, we adopt a coarse mesh made of 50 cells to be underresolved w.r.t. $\varepsilon$. Finally, the reference solutions are obtained by considering a converged solution with the standard splitting scheme. For all tests, this reference solution is compared with the standard splitting method as well as our scheme in two configurations for the asymptotic numerical flux $\psi$ : namely the HLL and LaxWendroff fluxes.

The first case involves the following source term: $S(u, v)=f(u)-v$ where $f(u)=\frac{1}{4}\left(e^{-u}-1\right)$. The initial data is $u(0, x)=u_{0}(x)=\sin (\pi(x-1))^{3}$ and $v(0, x)=f(u(0, x))$. The boundary conditions are $u(t, 0)=u(t, 2)=0$ and:

$$
\begin{aligned}
& v(t, 0)=u(t, 0)-\left(u_{0}-f\left(u_{0}\right)\right)(t), \\
& v(t, 2)=-u(t, 2)+\left(u_{0}+f\left(u_{0}\right)\right)(2-t) .
\end{aligned}
$$

The results for $t=0.5$ are given in figure 7 . We can see near the location of the discontinuity in $\varepsilon$ that the splitting scheme presents an important discrepancy with the reference solution. Our scheme clearly behaves better. It is important to note that its ability to take into account different choices of $\psi$ proves to be an important edge here. In fact, even in the region where $\varepsilon=1$ (far from the asymptotic regime), using a Lax-Wendroff flux for $\psi$ visibly improves the approximation.

The second case is similar to the previous one but with $f(u)=\frac{1}{4}\left(e^{u}-1\right)$. The main difference comes from the behavior of $u$ at the interface $x=1$ : the interface layer present in the former case does not exist here. The results are given at time $t=0.5$ in figure 8 . Once again, the splitting approach gives a poor approximation near the discontinuity while the HLL/Lax-Wendroff hybrid scheme performs well.

In the last case, the initial condition is the following Riemann problem:

$$
u_{0}(x)=\left\{\begin{array}{l}
-1 \text { if } x<0.8 \\
1 \text { otherwise }
\end{array}\right.
$$

The results are displayed on figure 9 at time $t=0.3$. Since the difficulty arises in the region where $\varepsilon$ is not small, the importance of the choice of $\psi$ is less predominant. Aside from that, our scheme still gives better results than the splitting. 

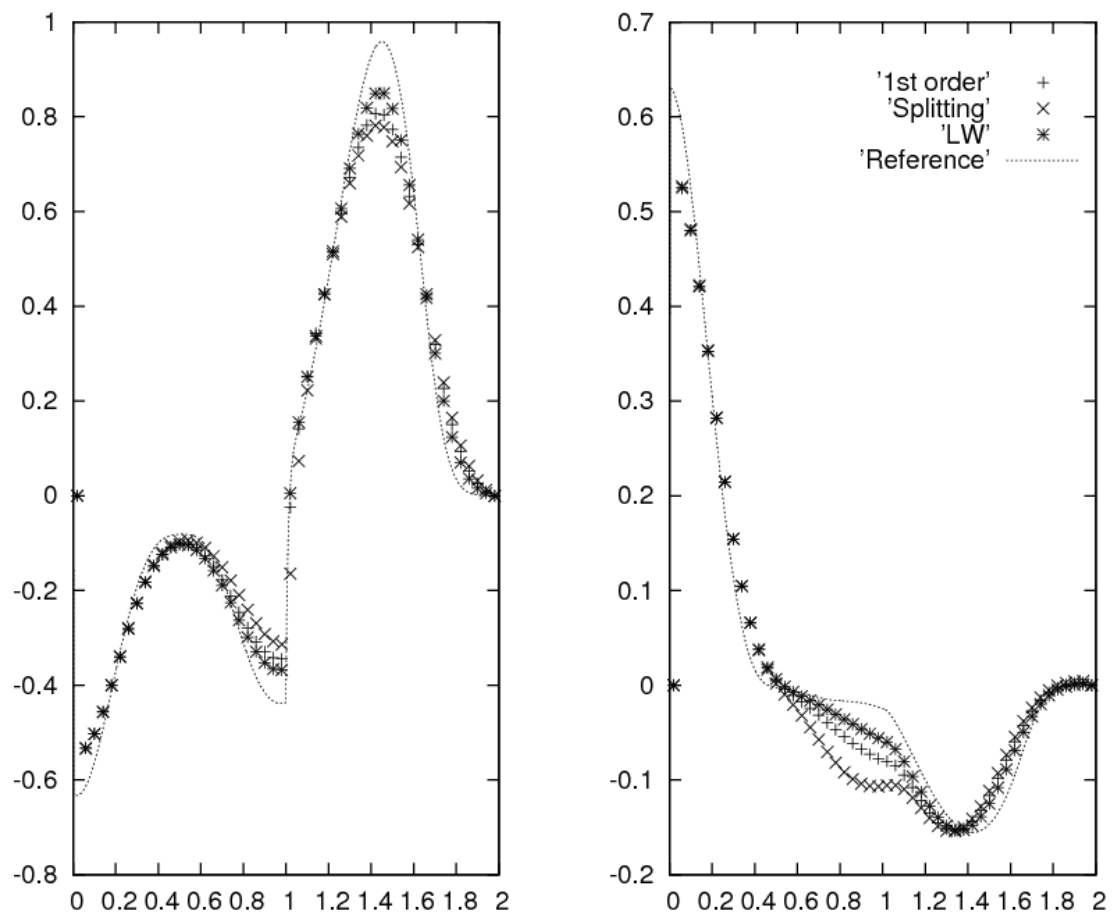

Figure 7: Variable $\varepsilon$ case 1: $\mathrm{u}(\mathrm{l})$ and $\mathrm{v}(\mathrm{r})$ at $t=0.5$. 

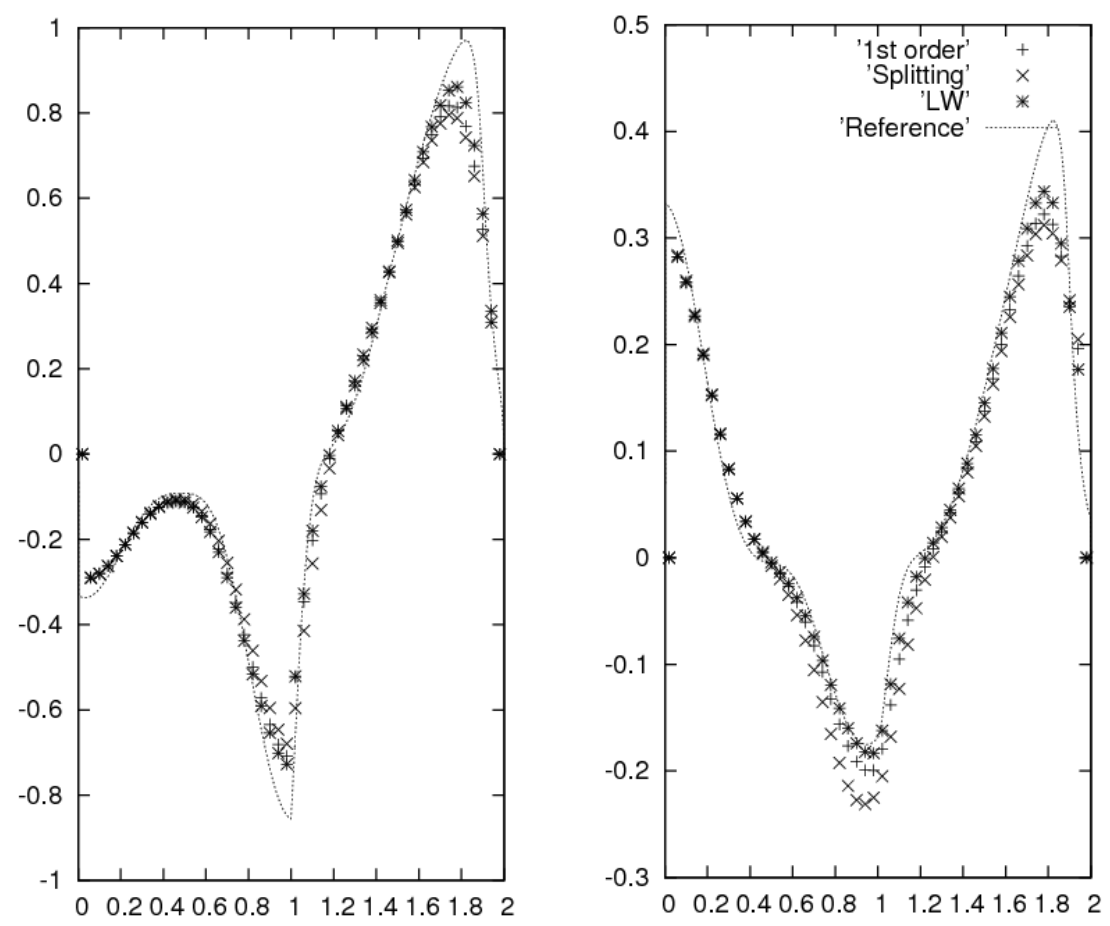

Figure 8: Variable $\varepsilon$ case 2: $\mathrm{u}(\mathrm{l})$ and $\mathrm{v}(\mathrm{r})$ at $t=0.5$. 


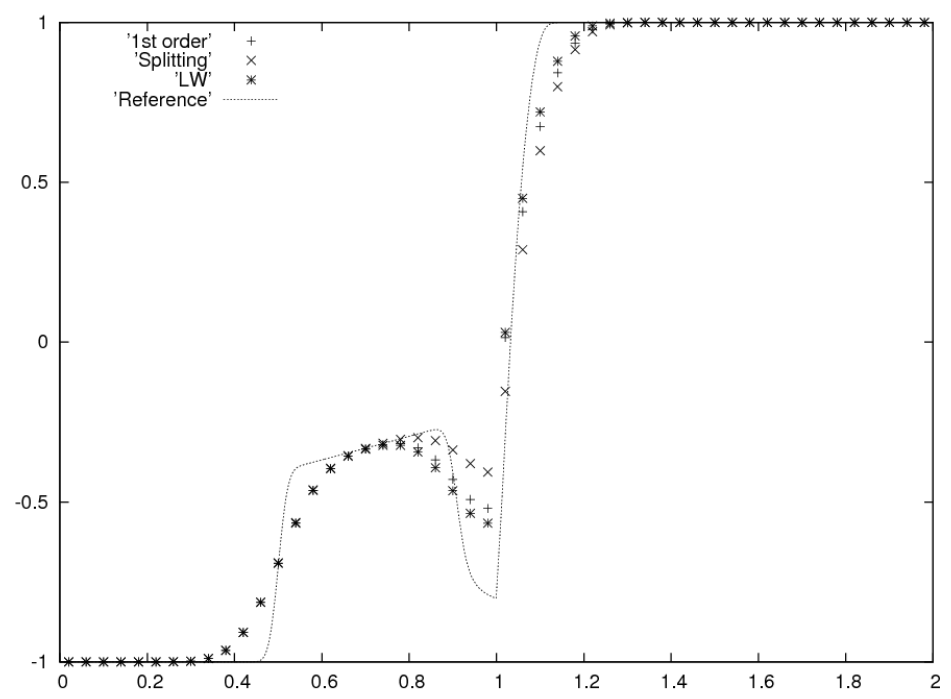

Figure 9: Variable $\varepsilon$ case $3: \mathrm{u}$ at $t=0.3$.

\subsection{Long time behavior}

In this last test-case, we investigate the long-time behavior of the schemes. We therefore consider the Telegraph equations (i.e. $f=0, g(u, v)=u$ and $a=1$ ) for a small $\varepsilon$ and compute the solutions for large times. This is a very challenging test-case all the more since $u$ is not governed by the limit equation (3) anymore. Instead, it degenerates into the solution of a diffusion equation (see [6] for instance). The results showed on figure 10 are obtained with $\varepsilon=5.10^{-3}$ and $t=40$ on a mesh made of 100 cells. The initial conditions are given by:

$$
(u, v)(0, x)=\left\{\begin{array}{l}
(-1,-1) \text { if } x<8 \\
(0,2) \text { otherwise }
\end{array}\right.
$$

Finally, the reference solution is obtained with a grid-converged long-time asymptoticpreserving scheme (see [7]). Obviously, neither the scheme introduced in this article nor the splitting strategy are designed for being able to recover the diffusive limit of this extreme test-case. Indeed, we immediately see on figure 10 that the splitting scheme gives results which are very far from the expected solution. Our scheme with the HLL choice for $\psi$ is better though it still predicts wrong results. On the other hand, the Lax-Wendroff flux for $\psi$ greatly improves the quality of the approximation and the discrepancy between the computed and the reference solution becomes acceptable. In fact, high-order choices for $\psi$ in- 

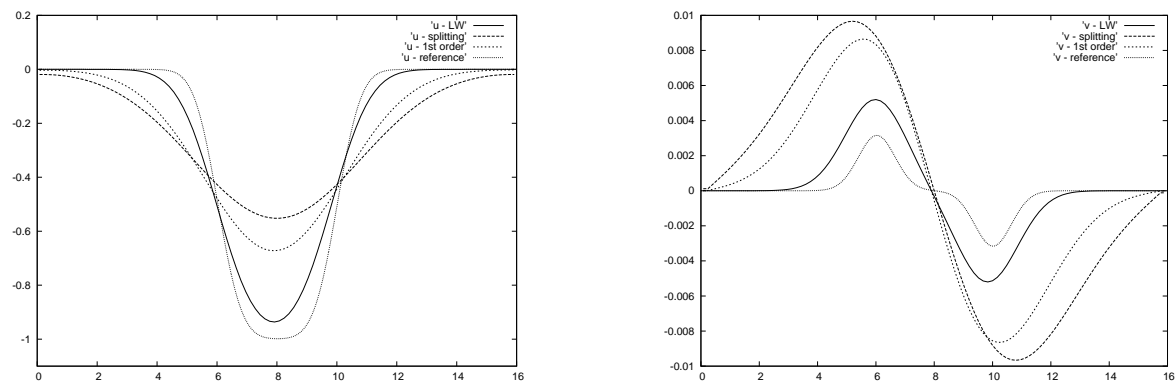

Figure 10: Long time $(t=40)$ behavior of the different schemes.

crease the range of validity of the scheme.

Let us finally point out that it seems incompatible to preserve both the relaxation and the diffusion asymptotics. Therefore, if simulations are to be carried out over a wide range of times, our scheme with a high-order choice of $\psi$ seems a good compromise.

\section{Conclusion}

In this paper, an asymptotic-preserving numerical scheme was proposed to approximate the solutions of (1)-(2). One of the main edges of this scheme is to fully control the numerical discretization of both asymptotic regimes. In particular, the main discrepancy between this scheme and the classical ones (such as the splitting technique) lies in the possibility to select any relevant scheme in the relaxation limit $(\varepsilon \rightarrow 0)$. Indeed, it is imposed by the usual techniques. This edge is important from several points of view. For instance, it allows to consider this scheme for code coupling. It is also crucial on extreme situations, as showed in the long-time simulation.

The scheme was proved to be robust and stable and to perform well on various numerical examples.

\section{References}

[1] A. Ambroso, C. Chalons, F. Coquel, and T. Galié. Interface model coupling via prescribed local flux balance. (07-3822), 2007.

[2] A. Ambroso, C. Chalons, F. Coquel, E. Godlewski, F. Lagoutière, P.-A Raviart, and N. Seguin. The coupling of homogeneous models for twophase flows. IJFV, 4(1):1-39, 2007.

[3] A. Ambroso, C. Chalons, F. Coquel, E. Godlewski, F. Lagoutière, P.-A. Raviart, and N. Seguin. Relaxation methods and coupling procedure. 56(8):1123-1129, 2008. 
[4] A. Ambroso, C. Chalons, and P.A. Raviart. A godunov-type method for the seven-equation model of compressible two-phase flow. Computers and Fluids, 54:67-91, 2012.

[5] D.S. Bale, R.J. Leveque, S. Mitran, and J.A. Rossmanith. A wave propagation method for conservation laws and balance laws with a spatially varying flux functions. SIAM J. Sci. Comput., 24:955-978, 2002.

[6] C. Berthon, P.-G. LeFloch, and R. Turpault. Late time/stiff relaxation asymptotic preserving approximations of hyperbolic equations. Math. of Comp., to appear.

[7] C. Berthon and R. Turpault. Asymptotic preserving hll schemes. Numerical Methods for Partial Differential Equations,.

[8] F. Bouchut. Nonlinear stability of finite volume methods for hyperbolic conservation laws and well-balanced schemes for sources. Frontiers in Mathematics. Birkhäuser Verlag, Basel, 2004.

[9] F. Bouchut, H. Ounaissa, and B. Perthame. Upwinding of the source term at interfaces for euler equations with high friction. Comput. Math. Appl., 53:361-375, 2007.

[10] C. Buet and S. Cordier. An asymptotic preserving scheme for hydrodynamics radiative transfer models. Numerische Mathematik, 108(2):199-221, 2007.

[11] C. Chalons, F. Coquel, E. Godlewski, P.A. Raviart, and N. Seguin. Godunov-type schemes for hyperbolic systems with parameter dependent source. the case of euler system with friction. Mathematical Models and Methods in Applied Sciences, 20(11):2109-2166, 2010.

[12] G.Q. Chen, C.D. Levermore, and T.P. Liu. Hyperbolic conservation laws with stiff relaxation terms and entropy. Comm. Pure Appl. Math., 47:787830, 1995.

[13] P. Degond, F. Deluzet, A. Sangam, and M.H. Vignal. An asymptotic preserving scheme for the euler equations in a strong magnetic field. J. Comput. Phys., 228:3540-3558, 2009.

[14] F. Filbet and S. Jin. A class of asymptotic-preserving schemes for kinetic equations and related problems with stiff sources. J. Comput. Phys., 229:7625-7648, 2010.

[15] G. Gallice. Positive and entropy stable godunov-type schemes for gas dynamics and mhd equations in lagrangian or eulerian coordinates. Numer. Math., 94(4):673-713, 2003.

[16] E. Godlewski and P.A. Raviart. Numerical approximation of hyperbolic systems of conservation laws, volume 118 of Applied Mathematical Sciences. Springer-Verlag, New York, 1996. 
[17] L. Gosse and Toscani. Asymptotic-preserving well-balanced scheme for the hyperbolic heat equations. C. R., Math., Acad. Sci. Paris, (334):337-342, 2002 .

[18] A. Harten, P.D. Lax, and B. Van Leer. On upstream differencing and Godunov-type schemes for hyperbolic conservation laws. SIAM Rev., 25(1):35-61, 1983.

[19] S. Jin, J.G. Liu, and L. Wang. A domain decomposition method for semilinear hyperbolic systems with two-scale relaxations. preprint, 2011.

[20] S. Jin and Z. Xin. The relaxation schemes for systems of conservation laws in arbitrary space dimensions. Comm. Pure Appl. Math., 48:235-276, 1995.

[21] R.J. LeVeque. Finite volume methods for hyperbolic problems. Cambridge Texts in Applied Mathematics. Cambridge University Press, Cambridge, 2002 .

[22] H. Mathis and N. Seguin. Model adaptation for hyperbolic systems with relaxation. Finite Volumes for Complex Application VI, pages 673-682, 2011.

[23] C. Parés. Path-conservative numerical schemes for nonconservative hyperbolic systems. Hyperbolic problems: theory, numerics, applications, Springer, (2):817-824, 2008.

[24] E.F. Toro. Riemann solvers and numerical methods for fluid dynamics. Springer-Verlag, Berlin, second edition, 1999. A practical introduction. 